\title{
Effort in isometric muscular contractions related to force level and duration ${ }^{1}$
}

\author{
JOSEPH C. STEVENS ${ }^{2}$ AND WILLIAM S. CAIN \\ JOHN B. PIERCE FOUNDATION LABORATORY, YALE UNIVERSITY
}

The degree of perceived force involved in squeezing a handgrip dynamometer is shown to grow as a power function of the force of isometric contraction and also as a power function of the duration of the squeeze. The exponent for force turns out to be more than twice that for duration. These two power functions are able to predict measurements of muscle endurance, i.e., of the maximum length of time that contraction of any constant level of force can be sustained.

It is a matter of everyday experience that the perceived magnitude of muscular exertion depends not only on the force of the exertion but also on how long the exertion lasts. The load that feels light when first hefted has a way of growing into an intolerable burden. This phenomenon relates to muscular fatigue, which is typically measured as a decline over time in strength, accuracy, or speed of performance, and not as the increased sense of effort experienced in submaximal exertion. It is, therefore, not known how the perceived intensities of sustained levels of force behave over time.

This paper raises the question: What functions describe the growth of apparent force with respect to the force level and to the duration of the exertion? In other words, what combinations of force level and duration produce the same momentary degree of perceived force? The answer was sought for one convenient type of muscular activity, namely isometric squeeze of a hand dynamometer. In brief, the procedure was to squeeze the dynamometer at various levels of force over various lengths of time and to assess, by numerical judgment, the degree of perceived force experienced at the end of the exertion.

Force of handgrip was examined earlier with a battery of psychophysical methods (J. C. Stevens \& Mack, 1959). Only brief (essentially phasic) responses were studied. Perceived force $\psi$ turned out to grow in proportion to the 1.7 power of exerted force $\varphi$ :

$$
\psi=\mathrm{k} \varphi^{1.7}
$$

or in the logarithmic form:

$$
\log \psi=\log \mathrm{k}+1.7 \log \varphi
$$

The exponent 1.7 implies the simple rule that the sensation of handgrip force increases by $100 \%$ whenever the actual force applied is increased by only $50 \%$. The same simple rule (or a close approximation to it) seems to apply to a wide variety of muscular activities. Table 1 is a brief summary of the research. Of course, Eq. 1 says nothing about how the sensation of force depends on duration, which is the main subject of the present study.

\section{Apparatus}

\section{METHOD}

The handgrip dynamometer was machined in to a hairpin shape from a single heavy piece of tempered aluminum. Force applied to the handle of the dynamometer was converted into an electrical signal by means of capacitor plates mounted oppostie each other on the inside surface of each arm of the handle. The plates formed part of an LC network. Any small movement of the arms relative to each other changed the area of overlap of the plates and, therefore, the capacitance. The capacitance constituted one leg of an ac bridge, and the rectified output of the bridge was fed to a dc recorder. At any given point along the arm, the relation between dc output voltage level and force applied to the handgrip was found to be stable. A squeeze of $100 \mathrm{lb}$ produced a 3-mm excursion of the arms at the particular point where they were to be grasped by the $S$ in the experiment.

The output from the bridge could also be fed directly to a simple meter consisting of a pointer and a single reference mark. This meter served as a monitor for the $S$. He was instructed to align the pointer with the marker on each trial. The amount of pressure on the dynamometer necessary for the alignment could be adjusted by means of an offset potentiometer connected to the meter. A trial began when a signal light was turned on; the $S$ brought the pointer to position as quickly as possible and maintained it there until the signal light went off.

The test sessions took place in a quiet sound-treated room. The S's chair was adjustable in height so that he was able to adopt a comfortable position for grasping the handle of the dynamometer with his preferred hand.

Table 1

Power Functions for Apparent Force

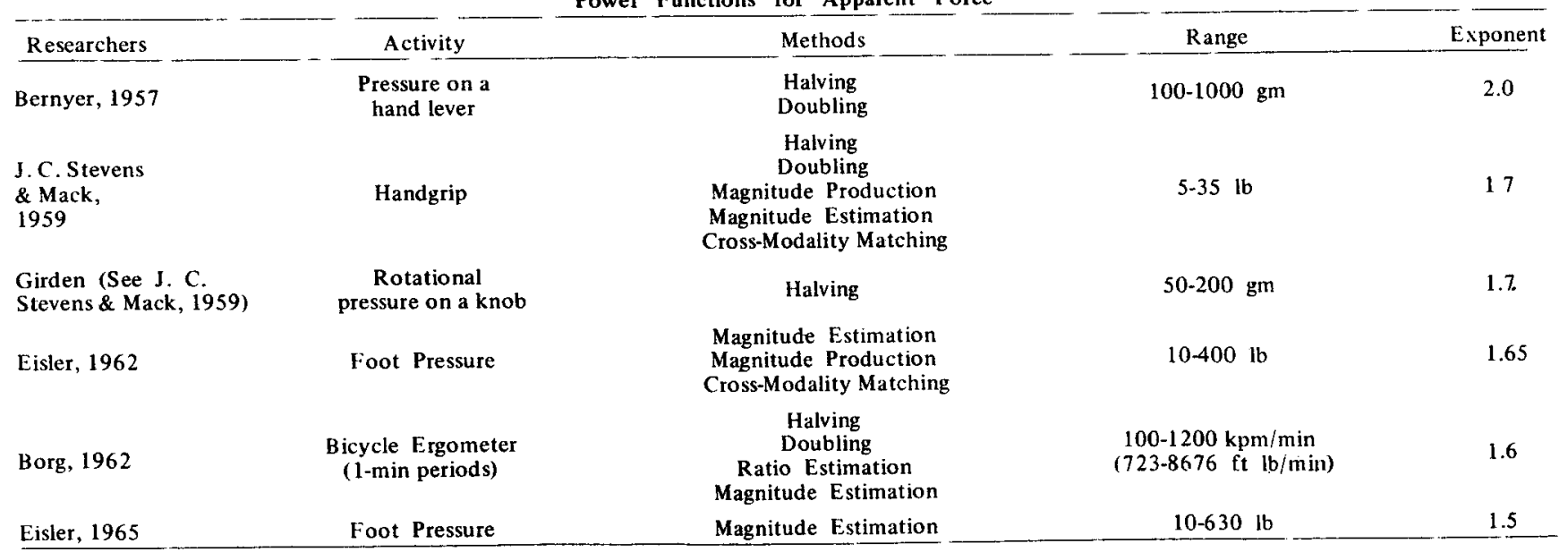




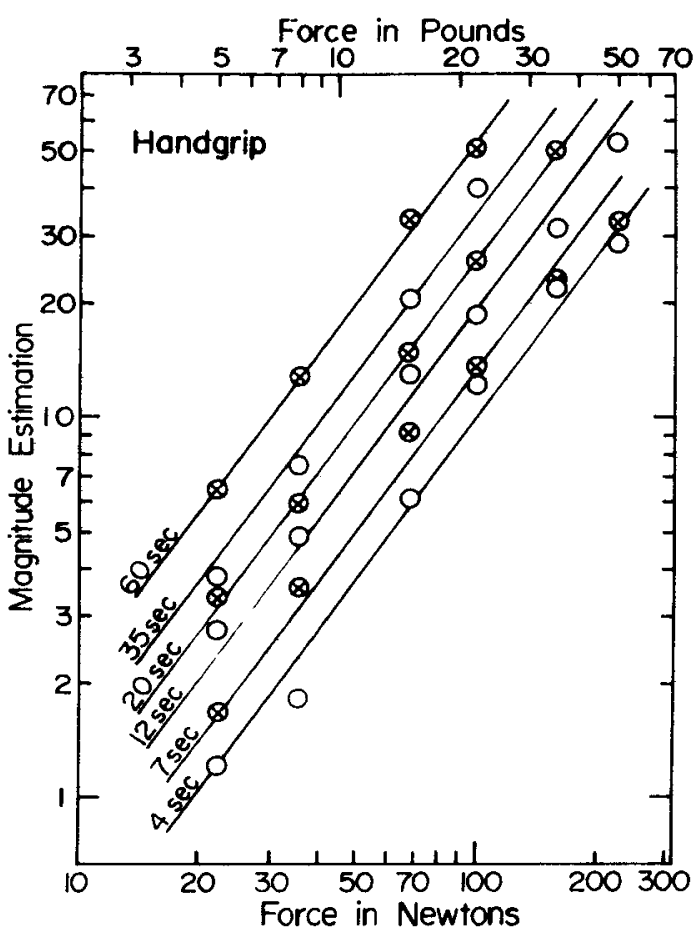

Procedure

A test session began with a brief demonstration of how to grasp the dynamometer, etc. This was practiced a few times by the $S$, until he was able to bring the pointer quickly up to the reference mark without seriously overshooting. Next, the $\mathrm{S}$ was told he would exert various amounts of force over various amounts of time. $\mathrm{H}_{\text {is }}$ task was to assess the apparent force experienced at the end of the trial, i.e., at the moment the signal light went out. On the first trial the $\mathrm{S}$ was asked to give a number of convenient size to stand for the degree of apparent force experienced (this number was not counted as an assessment); in subsequent trials, the $S$ assigned numbers in proportion to the terminal apparent force (the method of magnitude estimation).

A test session called for 31 different force-duration combinations (plus the squeeze at the start, not counted). The force levels ranged from 22 to $220 \mathrm{~N}$ ( 5 to $50 \mathrm{lb})$ and the durations from 4 to $60 \mathrm{sec}$. The order of the force-duration combinations was random in each test session.

Each of 18 male Ss (ages 18 to 37 ) served in two test sessions, separated by at least $24 \mathrm{~h}$. Because the squeezes sometimes aroused considerable fatigue and aftersensations in the hand and arm, fairly long intertrial rests were deemed desirable. No hard and fast rules were adopted. Some squeezes necessitated very short rests, others long rests. There were also different needs for rest from one $S$ to another,

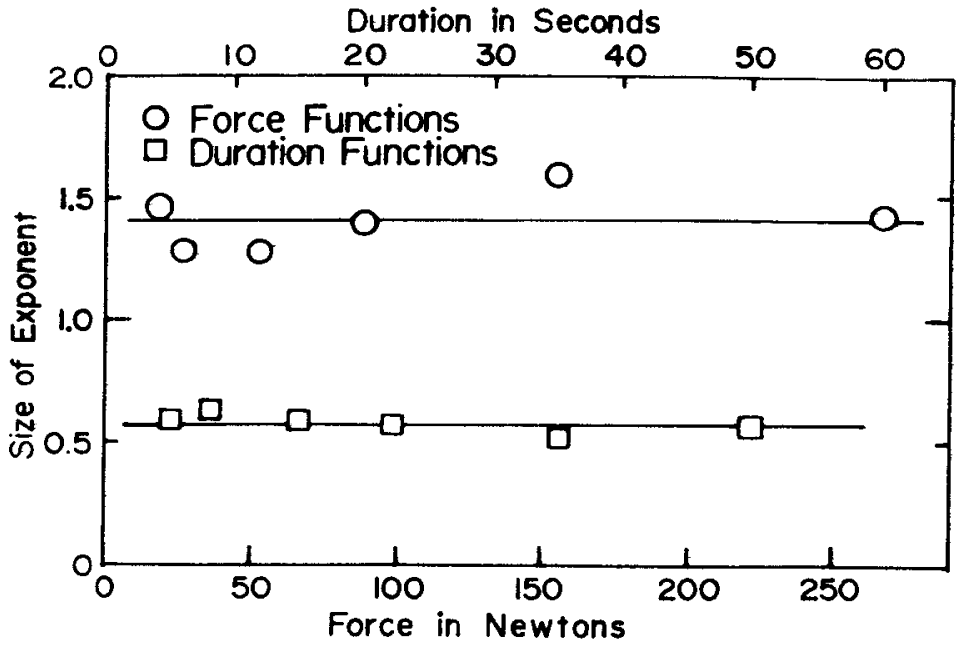

Fig. 1. (left) Perceived terminal force as a function of force of handgrip squeezes. The parameter is duration of the squeeze in seconds.

Fig. 2. (above) Exponents determined by the method of least squares using the geometric means of the magnitude estimates. depending on strength. The $S$ was repeatedly asked about fatigue and aftersensations. The average intertrial interval for all $S s$ and trials came to $2.5 \mathrm{~min}$. A typical session, including instructions, lasted about $2 \mathrm{~h}$.

\section{RESULTS AND DISCUSSION}

Power Functions for Force and Duration

For each force-duration combination the geometric mean was computed across Ss. In the log-log coordinates of Fig. 1, these means are plotted as a function of the force level. The six straight lines in Fig. 1, one for each duration, were fitted as a family. Under the assumption that the power function holds for any constant duration (i.e., that all of the functions in log-log coordinates are straight lines), the slope constant $\beta$ and the intercept constant $\log \mathrm{k}$ were determined by the method of least squares. From Fig. 2 it is apparent that, although the value of $\beta$ varied from 1.3 to 1.6 (average $=1.4$ ), the exact value does not seem to relate systematically to the duration of the contraction. Accordingly, all six lines in Fig. 1 were drawn with the same slope (1.4) and their relative positions $(\log \mathrm{k})$ determined by a second least-squares solution done under the assumption that $\beta$ is constant at 1.4 . It is clear from Fig. 1 that the positions of the functions vary systematically with duration, e.g., a given force level held for $60 \mathrm{sec}$ feels several times greater than the same level held for only 4 sec.

The same geometric means are plotted again in Fig. 3 as a function of the duration of the contraction. The data for each force level were fitted with a power function by a procedure like that applied in connection with Fig. 1. Figure 2 shows that the exponents (slopes) determined by least squares (average $=0.57$ ) do not vary systematically with force of contraction. All six lines in Fig. 3 were therefore drawn with the same slope $(0.57)$ and their relative positions $(\log \mathrm{k})$ determined by a second least-squares solution done under the assumption that $\beta$ is constant at 0.57 .

It follows from Fig. 1 and Fig. 3 that a single equation can be written to relate the perceived magnitude $\psi$ to the level $\varphi$ and duration $t$ of the contraction, namely:

$$
\psi=\mathrm{k} \varphi^{1.4} \mathrm{t}^{0.57}
$$

The relation between the two variables would seem to be simple. For any constant length of time, the same exponent (1.4) describes the growth of perceived force of contraction; for any constant degree of force, the same exponent (0.57) describes the growth of perceived force with, duration of contraction.

As a demonstration of the simplicity of this outcome, all 31 geometric means of the magnitude estimations have been plotted in Fig. 4 as a function of the product $F^{1.4} \mathrm{t}^{0.57}$. A straight line with slope equal to 1.0 describes the entire set of data. There is no clear tendency for the most deviant points to be related systematically to force level or duration; in other words, the scatter observed in Fig. 4 would appear to be random. 


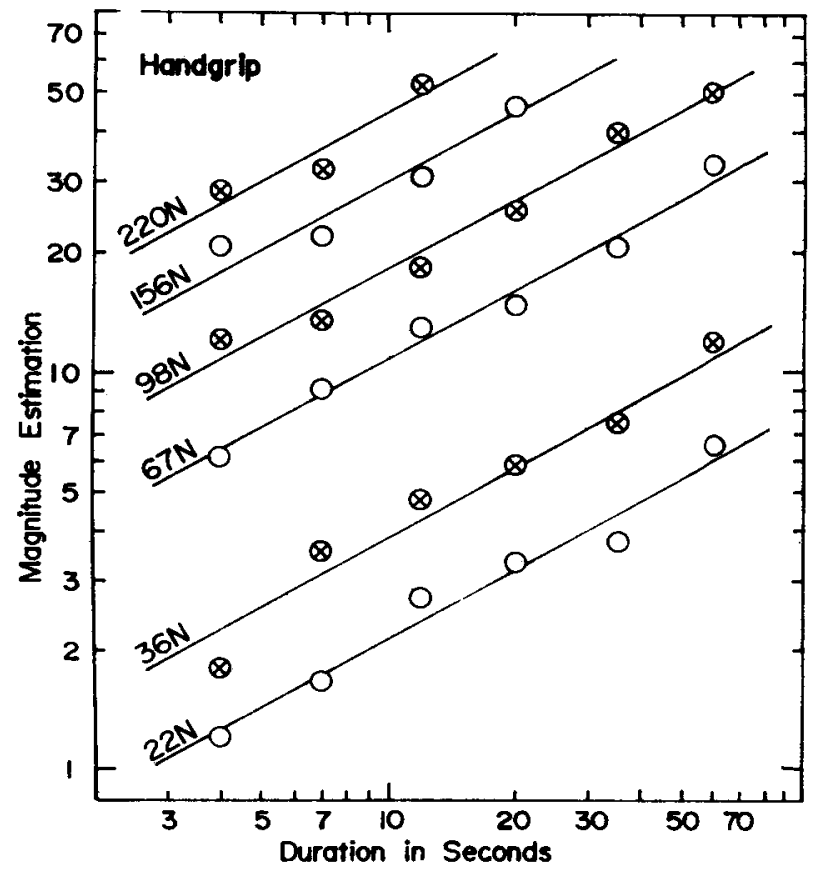

Fig. 3. Perceived terminal force as a function of duration. The parameter is the level of force in newtons.

\section{Effect of "Regression"}

The exponent 1.4 is smaller by about $12 \%$ than that found earlier for phasic contraction. The reason for this discrepancy is probably "regression bias," a very common type of bias in psychophysical measurement. (For the best account see S.S. Stevens \& Greenbaum, 1966.) Regression is, essentially, the tendency of the $S$ to constrict the range of whatever variables are placed under his control. With the method of magnitude estimation, this means that the range of numbers emitted is constricted, with the result that a misleadingly small exponent may be obtained. The earlier study of handgrip (J. C. Stevens \& Mack, 1959) brought out this point clearly. Magnitude estimation gave an exponent smaller than 1.7 , but the inverse procedure, magnitude production ( $S$ adjusts the force to match the number continuum), gave an exponent larger than 1.7. Similar results occurred for foot pressure (Eisler, 1962).

The effect of regression has often been shown to increase with the difficulty of the task imposed on the $S$, and the present task was surely difficult. Rests up to $10 \mathrm{~min}$ (average $2.5 \mathrm{~min}$ ) between judgments strain the memory and the attention. To show that under easier conditions a larger exponent would be obtained, a brief secondary experiment was conducted in which 10 Ss made 12 phasic (approximately 2-sec) squeezes (two for each of the six levels used before). Only short rest periods were needed, so the test session took only $5-10 \mathrm{~min}$. The results, in Fig. 5, determined a power function with slope (exponent) equal to 1.63 .

If regression operated to lower the exponent of the force functions by about $12 \%$, it must also have taken its toll of the exponent of the duration functions, presumably by approximately the same $12 \%$. Equation 3 may accordingly be revised (i.e., both exponents augmented by $12 \%$ to read:

$$
\psi=\mathrm{k} \varphi^{1.7} \mathrm{t}^{0.7}
$$

in order to take into account the probable extent of the effect of regression.

\section{Contours of Equal Perceived Force}

Equation 4 (or 3) can be rewritten to predict the combinations of force and duration of contraction that produce the same degree of terminal perceived force $\psi_{c}$

$$
\psi_{\mathrm{c}}=\mathrm{k} \varphi^{1.7} \mathrm{t}^{0.7}
$$

Since $\psi_{c}$ is a constant, Eq. 5 can be rearranged to read:

$$
\psi_{\mathrm{c}}=\mathrm{k} \varphi^{2.43_{\mathrm{t}}}
$$

Equation 6 is predicted equally well from Eq. 3 and Eq. 4 . That is to say, it is independent of the possible biasing effects of regression.

Relation of Equation 4 to Muscular Endurance

When a muscle or muscle group is contracted isometrically, a point will eventually come when the contraction can no longer be sustained at the required force level. Naturally, the greater the force level required, the sooner the muscle will reach the point of maximal endurance. It seems reasonable to suppose that, given any particular type of isometric contraction (e.g., handgrip), the sensation of force at the endurance point is independent of whether the endurance point is reached

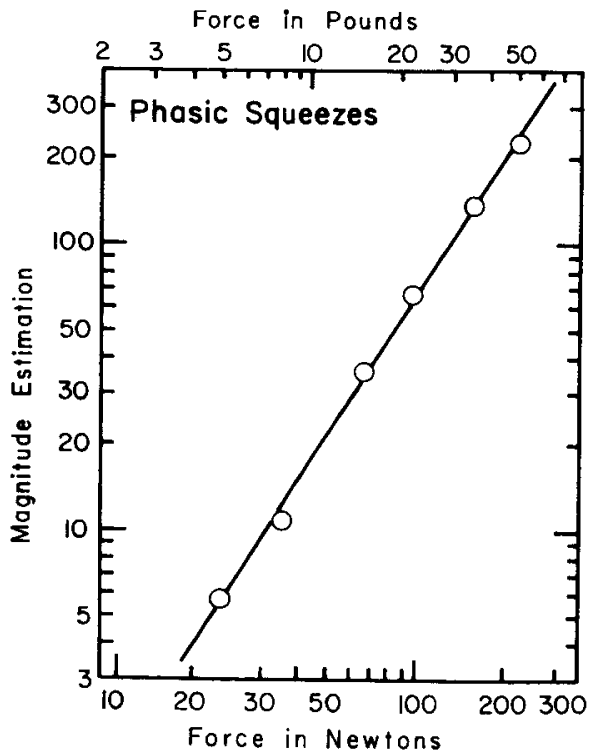

Fig. 5. Perceived force as a function of force of handgrip for brief squeezes of the dynamometer. 


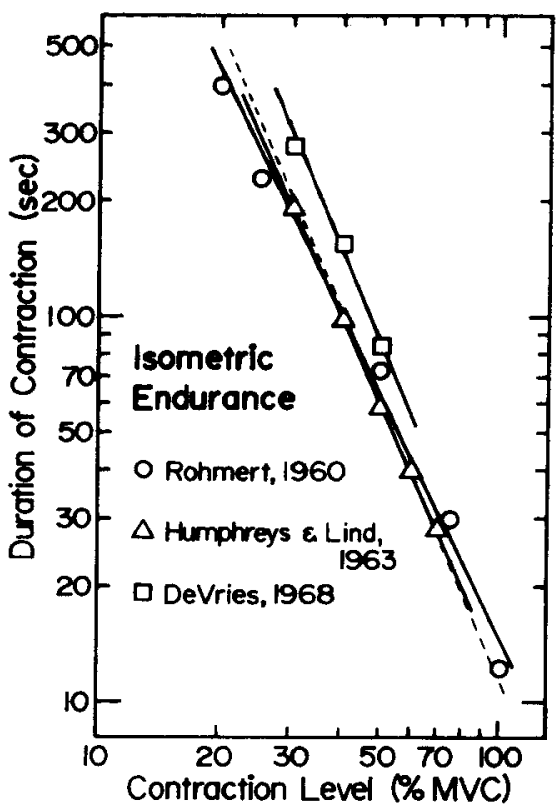

Fig. 6. Four isometric endurance functions relating the force of contraction (in percent of maximal voluntary contraction) and the length of time (in seconds) the contraction could be sustained. See text and Table 2 for explanation.

relatively fast by arduous contraction or more slowly by less arduous contraction or, in other words, that the perceived level of force is always the same at the limit of endurance. Let $\mathrm{K}$ stand for this constant perceived force. Then it follows from Eq. 6 that

$$
\mathrm{t}=\mathrm{K} / \varphi^{2.43}
$$

Equation 7 constitutes a prediction of the mathematical relation between duration and force level when a muscle group reaches the point of maximal endurance. Several Es have sought to measure that relation, and a large number of individual Ss have been tested on a variety of isometric muscle actions. Of course, individual persons and specific muscle groups vary widely in strength, but the relation between force and time to the endurance point shows some interesting invariances. These become apparent when the duration of the required contraction is plotted as a function of the percent of the maximum voluntary contraction (brief contraction) rather than simply as a function of the force of contraction per se.

Such a plot is Fig. 6, which summarizes the results from four endurance studies. See also Table 2. Taken together, the four studies cover both sexes, a sizeable number of $\mathrm{Ss}$, and a variety of muscle groups, but the results of all four can be fitted reasonably well with straight lines in log-log coordinates. The slopes and positions (intercepts) are fairly similar from one study to the other. (Since the present concern is with the slope of the endurance function, the apparent displacement of one line in Fig. 6 is not particularly relevant; nevertheless, it seems likely that differences in intercept might stem from varying definitions of, or conditions for the measurement of, maximum voluntary contraction.) The study by Monod and Scherrer (1957) is summarized in Fig. 6 by a straight line with slope equal to -2.42 , the exponent of the power function used by Monod and Scherrer to describe their results for four different types of muscular action. The 82 points plotted on their original graph stand for individual determinations and are too numerous to plot in our Fig. 6 . The various symbols plotted in Fig. 6 come from the other three studies and stand for average measurements; lines were fitted to these averages by the present authors.

The agreement between the average slope ( 2.33) of the endurance functions and the exponent $(-2.43)$ predicted in Eq. 7 is encouraging evidence that Eq. 3 is basically correct and that it applies to levels and durations far greater than those actually used in the present study. Like the contours of equal perceived force, the predicted isometric endurance function is not affected by the regression bias and is therefore equally well predicted from Eq. 3 and Eq. 4. Furthermore, the relative invariance of the slope of the endurance function from one muscle group to another suggests that the growth rate of perceived force with duration is also much the same from one muscle group to another. Of course, eventually it would be well to examine the growth rate, with respect to both duration and force, in a broad sample of muscular activities. The examination could be made with the methods used in the present experiment or, alternatively, by matching apparent force associated with one muscle group (unfatigued) to apparent force associated in another muscle group (fatigued to various degrees). Eisler (1962) has already demonstrated the usefulness of the matching of phasic contractions across different muscle groups.

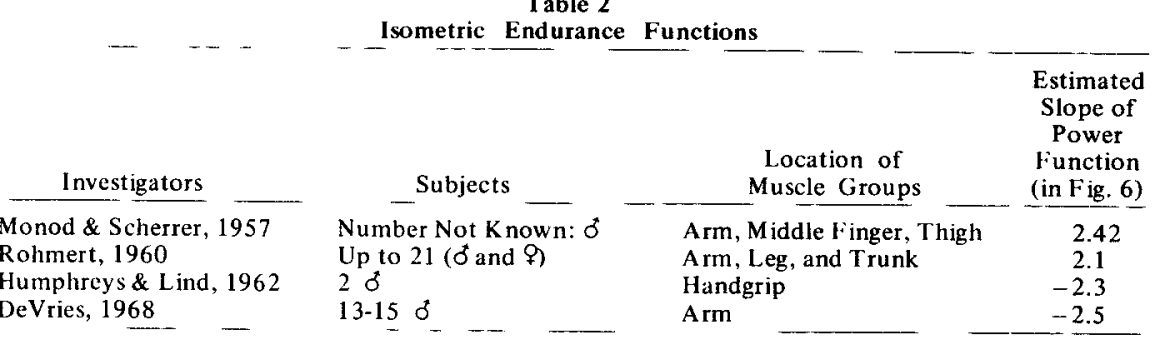

The relative invariance of the endurance functions in Fig. 6, which are largely based on averages across $S s$, suggests their potential use as a yardstick to evaluate individual differences in muscle performance or to assess the effects of pathology in muscle performance. The two Ss tested by Humphreys and Lind (1963), for example, gave curves under normal conditions that were distinctly different from those given under artificial occlusion of the forearm circulation. Occlusion barely altered the normal maximum duration of the strong short contractions but greatly reduced the normal maximum duration of the longer contractions. This finding suggests that occlusion might also alter the equal sensation contours at submaximal levels, perhaps by increasing the size of the exponent governing the growth of apparent force as a function of time

\section{Equal Subjective Force Over Time}

The results reported here were obtained for forces whose physical level was always maintained constant over time. Under that condition, the subjective level of force was seen to be ever increasing. An implication of this finding is that under the instruction to maintain a constant subjective force level over time, the $S$ will have to exert an ever diminishing degree of physical force level over time. This was the result obtained by Eason (1959) for an initial force level of $30 \mathrm{lb}$ and by the present authors (unpublished data) for each of a number of initial force levels. The application of the two methods (maintenance of constant physical force level and maintenance of constant subjective force level) holds out the attractive promise of greatly broadening our picture of the course of muscular sensations and, therefore, of the nature of muscular fatigue.

\section{REFERENCES}

BERNYER, G. Discussion in symposium: Le probleme de la mesure. Proceedings of the 15 th International Congress of Psychology (Brussels), 1957, 99.100.

BORG, G. A. V. Physical performance and perceived exertion. Studia Psychologica et Paedagogica, 1962, 11, 1.64.

DeVRIES, H. A. Method for evaluation of muscle fatigue and endurance from electromyographic 
fatigue curves. American Journal of Physical Medicine, 1968, 47, 125-135.

EASON, R. G. The surface electromyogram (EMG) gauges subjective effort. Perceptual \& Motor Skills, 1959, 9, 359-361.

EISLER, H. Subjective scale of force for a large muscle group. Journal of Experimental Psychology, 1962, 64, 253-257.

EISLER, H. The ceiling of psychophysical power functions. American Journal of Psychology, 1965, 78, 506-509.

HUMPHREYS, P. W., \& LIND, A. R. The blood low through active and inactive muscles of the forearm during sustained hand-grip contractions. Journal of Physiology, 1963
$166,120-135$.

MONOD, H., \& SCHERRER, J. Capacité de travail statique d'un groupe musculaire synergique chez l'homme. Comptes Rendus de la Société de Biologie, 1957, 151, 1358-1362.

ROHMERT, W. Ermittlung von Erholungspausen für statische Arbeit des Menschen. Internationale Zeitschrift für Angewandte

Physiologie einschliesslich Arbeitphysiologie, $1960,18,123-164$.

STEVENS, J. C., \& MACK, J. D. Scales of apparent force. Journal of Experimental Psychology, 1959, 58, 405-413.

STEVENS, S. S., \& GREENBAUM, H. B

Regression effect in psychophysical judgment.
Perception 439-446.

\& Psychophysics, 1966, 1

\section{NOTES}

1. This reserach was supported by Contract F44620-67-C-0017 with the U.S. Air Force Office of Scientific Research and was performed while the second author was a U.S. Public Health Service postdoctoral fellow.

2. Address: John B. Pierce Foundation Laboratory and Yale University, New Haven, Connecticut 06519.

(Accepted for publication December 15, 1969.) 